\title{
A Powertrain LQR-Torque Compensator with Backlash Handling
}

\author{
P. Templin ${ }^{1 *}$ and B. Egardt ${ }^{2}$ \\ 1 Volvo Powertrain Corporation, Göteborg, 40508 - Sweden \\ 2 Chalmers University of Technology, Göteborg, 41296 - Sweden \\ e-mail: peter.templin@volvo.com - bo.egard @chalmers.se \\ * Corresponding author
}

\begin{abstract}
Résumé - Régulation par LQR d'une chaîne cinématique automobile avec jeu : simulation et étude expérimentale - Cet article présente un dérivé de régulateur anti-saccades LQR destiné à une chaîne cinématique automobile. La dérivée par rapport au temps du couple de l'arbre de transmission, qui est en relation étroite avec la saccade du véhicule, est utilisée en tant que donnée de sortie de système virtuel et régulée à zéro. Ainsi, le régulateur ne nécessite pas de modèle de référence destiné à la génération de trajectoires de référence pour l'évaluation de la loi de régulation. Le régulateur agit en tant que compensateur de couple pour la demande de couple du conducteur que les données de sortie du régulateur suivent asymptotiquement. Les propriétés du régulateur sont étudiées et le comportement est illustré par des exemples de simulation et vérifiées à l'aide d'expériences sur un camion à usage industriel.
\end{abstract}

Abstract - A Powertrain LQR-Torque Compensator with Backlash Handling: Simulation and Experimental Study - This paper derives an LQR anti-jerk controller for an automotive driveline. The time derivative of the drive shaft torque, which is closely related to the vehicle jerk, is used as a virtual system output and regulated to zero. Thereby, the controller does not need a reference model for generation of reference trajectories for the control law evaluation. The controller acts as a torque compensator for the driver's torque demand which the controller output asymptotically follows. The properties of the controller are discussed and the behavior is illustrated by simulation examples and verified with experiments on a heavy duty truck. 


\section{INTRODUCTION}

The main task of an automotive powertrain is to deliver a tractive torque at the wheels, according to the driver's torque request. Since the powertrain is naturally a lightly damped oscillatory system with inevitable backlash, care needs to be taken during rapid transients. The perceived vehicle drivability is affected in a negative way if oscillations are induced in the vehicle tractive force that cause variations in the vehicle acceleration, known as shuffle or vehicle jerking. Similarly, backlash traversals giving rise to torque impulses, so called shunt, should be avoided.

The problem to damp powertrain oscillations has received a lot of attention in the literature, e.g. [1-6]. Most of these contributions are based on classical linear control techniques, including Linear Quadratic (LQ) design. Recently, [7] presented an LQR-based controller for damping driveline oscillations. The developed controller has the attractive feature of fitting very well into the archetypical heavy truck engine control system of today, being based on torque control. Thus, the controller can easily be incorporated as an additional torque compensator. The controller damps driveline oscillations by compensating the driver's engine torque demand, which is asymptotically tracked. An important property of the design is that no model based reference trajectories need to be generated.

Gear play and other backlash in the powertrain present an additional difficulty. In order to avoid unpleasant effects caused by the impact after a backlash traversal, including possible excitation of oscillatory modes, the backlash needs to be traversed gently. Obviously, this has to be traded against loss of responsiveness to torque requests. Backlash compensation has been treated by many authors. Applications to automotive powertrains can be found in e.g. $[1,8]$ and references therein. The state-of-the-art in production vehicles is to use simple but carefully tuned compensation schemes to avoid traversing the backlash too rapidly.

The present contribution has two main objectives. The first one is to extend the LQ-based damping controller presented in [7] with a feature to compensate for backlash. The idea is to limit the relative speed between the engine and the wheels during the backlash transition, by limiting the engine torque setpoint. The second objective is to report on an experimental evaluation of the combined powertrain controller applied to a heavy truck.

The paper is organized as follows. Section 2 presents the powertrain model used. Section 3 summarizes the control design requirements, followed by a description of the actual control design of the torque compensator and the backlash controller. Experimental results are summarized and discussed in Section 5. Some concluding remarks end the paper.

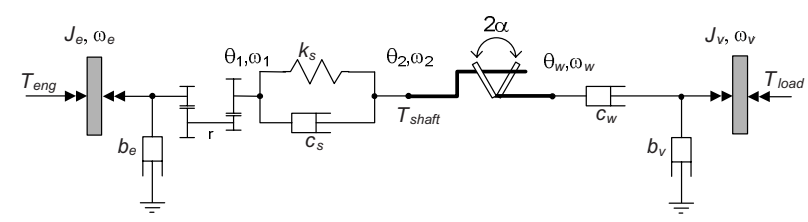

Figure 1

Driveline model with backlash.

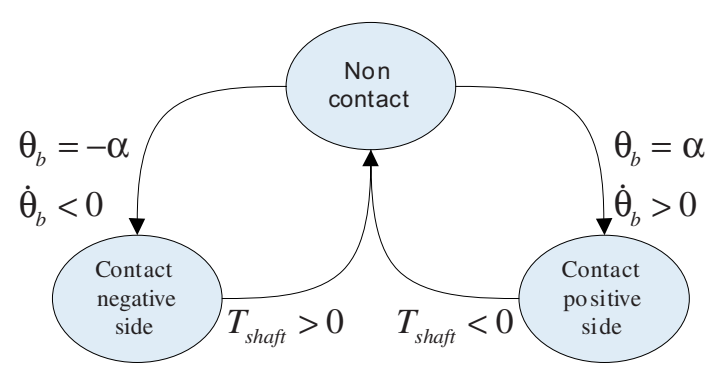

Figure 2

Model of mode transition conditions. $\theta_{b}$ is the backlash angle position and $T_{\text {shaft }}$ is the drive shaft torque.

\section{POWERTRAIN MODEL}

The powertrain model used in the present work is intended for parameter identification and control design using the measurement signals normally available in a powertrain control unit. The piecewise linear fourth order model, Figure 1, has been discussed in more detail in [9] and a motivation for the backlash description is given in [10].

The nonlinearity introduced by the backlash effectively decouples the engine from the wheels during the transition of the backlash. It is therefore possible to define three modes of the model, Figure 2. The powertrain can be in contact mode either on the negative or on the positive drive side, or it can be in non-contact mode during the backlash transition. The conditions for changing modes are given in the figure. The main variables for the changes are the drive shaft torque and the current position in the backlash. The drive shafts can not transmit positive (negative) torque when the system is in contact on the negative (positive) side.

The model in Figure 1 has two input signals, engine torque $T_{\text {eng }}$ and road load $T_{\text {load }}$. Engine speed $\omega_{e}$ and vehicle speed $\omega_{v}$ are measurable output signals and also model state variables. The third state is the drive shaft torsion angle $\theta_{s}=\theta_{1}-\theta_{2}$ and the last is the backlash position angle $\theta_{b}=\theta_{2}-\theta_{w}$. The derivative of the drive shaft torque $T_{\text {shaft }}$ and the backlash angle $\theta_{b}$ are defined as model outputs. The backlash size is defined to be $2 \alpha$ so that the backlash position angle $\theta_{b}$ is limited by $-\alpha \leq \theta_{b} \leq \alpha$.

The engine is described as an ideal torque source $T_{\text {eng }}$ with a mass-moment of inertia $J_{e}$ and viscous friction $b_{e}$. 


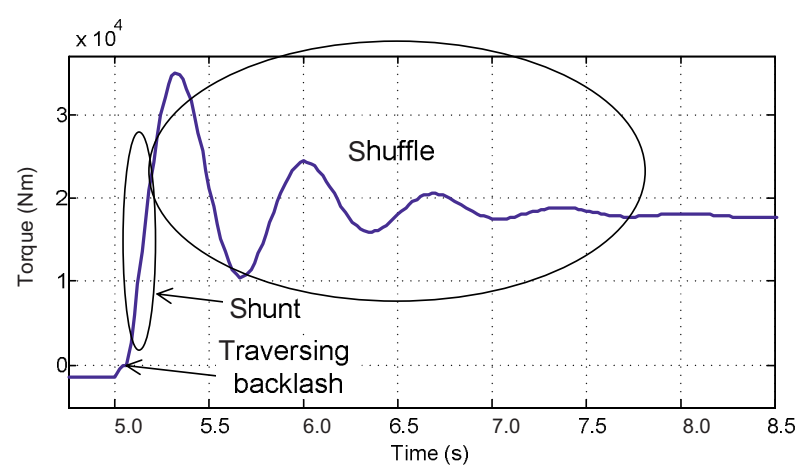

Figure 3

Simulation of the drive shaft torque after an engine torque step.

The gearbox ratio together with the final gear is described by the ratio $r$. It is assumed that the driveline main flexibility is in the drive shafts, represented by $k_{s}$ and $c_{s}$.

Wheel slip is often neglected for simplicity. In this approach it is represented by the damper $c_{w}$. The vehicle inertia is denoted $J_{v}$ with viscous friction term $b_{v}$. The road load is described by the exogenous signal $T_{\text {load }}$. Wheel speed $\omega_{w}$ is not a model state variable since the wheel slip is included but it can be defined as an extra model output if needed.

The state space representation (1) - (2) can be derived from the laws of motion and the properties of the dampers with $c^{\prime}=\frac{c_{w}}{c_{w}+c_{s}}$ defined for convenience.

Figure 3 shows a simulation of a pedal tip-in maneuver by the driver that illustrates the shunt and shuffle-phenomenon. Initially, the system is in contact mode on the negative drive side. Then follows the traversing of the backlash and, finally, the system is in contact on the positive drive side. During the transition of the backlash, the engine accelerates freely which makes the relative speed compared to the wheels

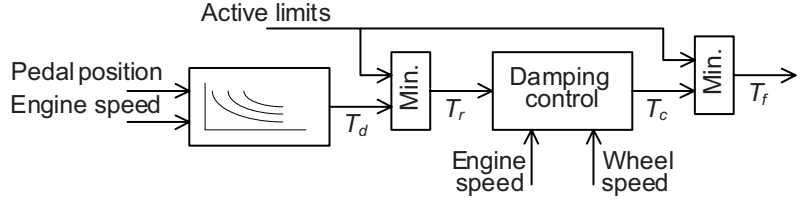

Figure 4

Engine management system torque control structure. $T_{d}$ is the driver's torque demand, $T_{r}$ is the torque reference set point, $T_{c}$ is the damping control compensated value and $T_{f}$ is the torque used by the lower level fueling strategies.

higher. A high impact speed amplifies the forthcoming oscillations and may also be clearly audible to the driver. It is therefore essential that a controller monitors the transition conditions between the the modes.

The following section will discuss requirements on a damping controller for the described system.

\section{CONTROLLER REQUIREMENTS}

The main requirements on the powertrain controller are performance related, and as described in Introduction the emphasis is on drivability. The drivability requirement is here basically translated into requirements on damping of driveline oscillations and smooth traversal of the driveline backlash, while still providing a quick response to driver torque demands.

In addition to the performance related demands on the controller, it is argued in [7] that the controller should fit into the existing control structure of the diesel engine control unit. The resulting control structure is depicted in Figure 4, where it can be seen that the main source for engine torque requests is the driver. The accelerator pedal position is translated into an engine torque demand. This is then compared to the set of active torque limitations and compensated

Contact mode:

$$
\left(\begin{array}{c}
\dot{\theta}_{s} \\
\dot{\omega}_{e} \\
\dot{\omega}_{v} \\
\dot{\theta}_{b}
\end{array}\right)=\left(\begin{array}{cccc}
-\frac{k_{s} c^{\prime}}{c_{w}} & \frac{c^{\prime}}{r} & -c^{\prime} & 0 \\
-\frac{k_{s} c^{\prime}}{J_{e} r}-\frac{c^{\prime}\left(\frac{c_{s}}{r^{2}}+\frac{c_{s} b_{e}}{c_{w}}+b_{e}\right)}{J_{e}} & \frac{c_{s} c^{\prime}}{r J_{e}} & 0 \\
\frac{k_{s} c^{\prime}}{J_{v}} & \frac{c_{s} c^{\prime}}{J_{v} r} & -\frac{c^{\prime}\left(\frac{b_{v} c_{s}}{c_{w}}+c_{s}+b_{v}\right)}{J_{v}} & 0 \\
0 & 0 & 0 & 0
\end{array}\right)\left(\begin{array}{c}
\theta_{s} \\
\omega_{e} \\
\omega_{v} \\
\theta_{b}
\end{array}\right)+\left(\begin{array}{c}
0 \\
\frac{1}{J_{e}} \\
0 \\
0
\end{array}\right) T_{\text {eng }}+\left(\begin{array}{c}
0 \\
0 \\
-\frac{1}{J_{v}} \\
0
\end{array}\right) T_{\text {load }}
$$

Non-contact mode:

$$
\left(\begin{array}{c}
\dot{\theta}_{s} \\
\dot{\omega}_{e} \\
\dot{\omega}_{v} \\
\dot{\theta}_{b}
\end{array}\right)=\left(\begin{array}{cccc}
-\frac{k_{s}}{c_{s}} & 0 & 0 & 0 \\
0 & -\frac{b_{e}}{J_{e}} & 0 & 0 \\
0 & 0 & -\frac{b_{v}}{J_{v}} & 0 \\
\frac{k_{s}}{c_{s}} & \frac{1}{r} & -1 & 0
\end{array}\right)\left(\begin{array}{c}
\theta_{s} \\
\omega_{e} \\
\omega_{v} \\
\theta_{b}
\end{array}\right)+\left(\begin{array}{c}
0 \\
\frac{1}{J_{e}} \\
0 \\
0
\end{array}\right) T_{\text {eng }}+\left(\begin{array}{c}
0 \\
0 \\
-\frac{1}{J_{v}} \\
0
\end{array}\right) T_{\text {load }}
$$


by the damping/backlash controller to be presented shortly. Finally, the active torque limits are applied again. The first application of the limits ensures that the damping/backlash controller always has a realistic setpoint. The impact of the second is minimized by a tuning of the controller that minimizes overshoots at requested torque rises. A consequence of the chosen control structure is that the compensated torque $T_{c}$ must approach the setpoint $T_{r}$ asymptotically.

It is desirable that the complete controller should be easy to tune. Since the controller is model based, much of the tuning is done implicitly by fitting a model to the specific powertrain. Additional tuning can often be made with a small number of parameters that affect the closed loop properties in an intuitive way. This will be illustrated in the section on experimental evaluation.

\section{CONTROL DESIGN}

The control design is based on the presented piecewise linear model of the system. The first section describes the observer that is is used to gain state vector information and to detect system mode changes. Then a linear LQR torque compensator for the contact mode is derived. Finally, an optimization based strategy is applied for the traversal of the backlash.

\subsection{State Observer}

The controller relies on full state information for calculation of the engine torque request, but only the engine and vehicle speed are measurable outputs. It is furthermore not possible to measure the backlash position to determine if the system is in contact or non-contact mode.

An observer for the contact mode can not be based on the state space description (1) directly with the available measurement signals. The system is not observable. But the fourth order model may be reduced to third order since the backlash angle is constant in contact mode. Then the state vector is observable using either both available measurements or the engine speed only, if the quality of the vehicle speed measurement is poor. In this work the Loop Transfer Recovery (LTR)-methodology is used for the contact mode observer design $[2,11]$. The observer outputs are the drive shaft twist and torque, which are used for the control law evaluation and state mode transitions, respectively.

When the system enters the backlash mode, the engine and the wheels are effectively decoupled and the system observability is lost. In [8], this is solved by using engine and wheel angle measurements, but these signals are not available in most standard trucks. This means that state information can only be estimated by using a system simulation in this mode $[9,12]$.
With the described observer, using the LTR-based design for the contact mode, a system simulation for the noncontact mode and the mode transition conditions from Figure 2, the torque compensator has all the information it needs for the control law evaluation.

\subsection{Torque Compensator}

The contact mode is characterized by that the backlash angle is constant, which essentially means that the fourth state equation row of (1) is not active in this mode. The torque compensator is therefore derived using (1) with the backlash angle state removed, so the state vector is here defined as $x_{a}=\left(\theta_{s}, \omega_{e}, \omega_{v}\right)$. The upper left $3 \times 3$-part of the system matrix in (1) is denoted $A_{a}$ and the corresponding input matrix $B_{a}$. The drive shaft torque can be defined as an output.

$$
\begin{aligned}
T_{\text {shaft }}(t) & =\left[c^{\prime} k_{s} \frac{c^{\prime} c_{s}}{r}-c^{\prime} c_{s}\right] x_{a}(t) \\
& =C_{a} x_{a}(t)
\end{aligned}
$$

The main task for the torque compensator is to damp driveline oscillations (the shuffle-part in Fig. 3), which in this context is interpreted as regulating the time derivative of the drive shaft torque to zero. The derivative of the drive shaft torque is therefore defined as a model output and included in the performance index. An advantage with this choice is that no state reference trajectories are needed for the implementation of the final control law since the reference value for the drive shaft torque derivative is always zero. The control signal should always be included in a performance index as a mean to balance performance and control effort. In this case however, a requirement of the controller is to asymptotically follow the driver's torque request. A solution to this is to include the difference between the driver's torque demand and the controller output in the performance index.

Integral action is introduced by extending the model by a fourth state $x_{u}$ that integrates the difference between the driver's torque demand and the controller output. This ensures that the controller output asymptotically follows the driver's torque demand. The performance index can then be written

$$
J=\int_{0}^{t_{f}}\left(\frac{1}{2} y^{T}(t) Q y(t)+\frac{1}{2}\left(u(t)-u_{r}\right)^{2}\right) d t
$$

with the system outputs $y^{T}(t)=\left[\dot{T}_{\text {shaft }}(t) x_{u}(t)\right]$, a diagonal positive semidefinite $2 \times 2$ weighting matrix $Q=\operatorname{diag}\left(q_{1}, q_{2}\right)$, the controller output $u(t)=T_{c}$ and the driver's engine torque demand $u_{r}=T_{r}$ (see Fig. 4).

Before the optimal control law that minimizes (4) can be derived by standard methods it needs to be re-formulated. Define the steady state solution $x_{a}^{r}$ such that

$$
A_{a} x_{a}^{r}+B_{a} u_{r}=0 \Rightarrow x_{a}^{r}=-A_{a}^{-1} B_{a} u_{r}
$$


Define a new set of state variables $\zeta$ and a new control variable $v$.

$$
\begin{aligned}
& \zeta(t)=\left[\begin{array}{c}
x_{a}(t)-x_{a}^{r} \\
x_{u}(t)
\end{array}\right] \\
& v(t)=\left(u(t)-u_{r}\right)
\end{aligned}
$$

Desired outputs are the derivative of the drive shaft torque (3) and the fourth state $x_{u}$. The transformed system then becomes

$$
\begin{gathered}
\dot{\zeta}=\left[\begin{array}{cc}
A_{a} & 0 \\
0 & 0
\end{array}\right]\left[\begin{array}{c}
x_{a}-x_{a}^{r} \\
x_{u}
\end{array}\right]+\left[\begin{array}{c}
B_{a} \\
1
\end{array}\right]\left(u-u_{r}\right) \\
\hat{=} A \zeta(t)+B v(t) \\
T_{\text {shaft }}(t)=C_{a} x_{a}(t) \Rightarrow \\
\dot{T}_{\text {shaft }}(t)=C_{a} A_{a} x_{a}(t)+C_{a} B_{a} u(t)= \\
=C_{a} A_{a}\left(x_{a}(t)-x_{a}^{r}(t)\right)+C_{a} B_{a}\left(u(t)-u_{r}\right) \\
y=\left[\begin{array}{c}
\dot{T}_{\text {shaft }} \\
x_{u}
\end{array}\right]=\left[\begin{array}{cc}
C_{a} A_{a} & 0 \\
0 & 1
\end{array}\right] \zeta+\left[\begin{array}{c}
C_{a} B_{a} \\
0
\end{array}\right] v \\
\hat{=} C \zeta+D v
\end{gathered}
$$

The minimization of (4) can now be written

$$
\begin{aligned}
& \min \int_{0}^{t_{f}}\left(\frac{1}{2} y^{T}(t) Q y(t)+\frac{1}{2} v(t)^{2}\right) d t= \\
& =\min \int_{0}^{t_{f}} \frac{1}{2}\left[\begin{array}{ll}
\zeta^{T} & v
\end{array}\right]\left[\begin{array}{cc}
C^{T} Q C & C^{T} Q D \\
D^{T} Q C & 1+D^{T} Q D
\end{array}\right]\left[\begin{array}{c}
\zeta \\
v
\end{array}\right] d t \\
& \hat{=} \min \int_{0}^{t_{f}} \frac{1}{2}\left[\begin{array}{ll}
\zeta^{T} & v
\end{array}\right]\left[\begin{array}{c}
Q_{\zeta} S \\
S^{T}
\end{array}\right]\left[\begin{array}{c}
\zeta \\
v
\end{array}\right] d t
\end{aligned}
$$

which is solved by using the Riccati-equation and forming a state feedback control law.

$$
\begin{aligned}
A^{T} P+P A- & (P B+S) R^{-1}\left(B^{T} P+S^{T}\right)+Q_{\zeta}=0 \\
K= & R^{-1}\left(B^{T} P+S^{T}\right)=\frac{\left(B^{T} P+S^{T}\right)}{1+D^{T} Q D} \\
K_{(1 \times 4)=} & {\left[K_{a(1 \times 3)} K_{u(1 \times 1)}\right] } \\
v(t)= & -K \zeta(t)=-K_{a}\left(x_{a}(t)-x_{a}^{r}\right)-K_{u} x_{u} \Rightarrow \\
u(t)= & -K_{a} x_{a}(t)-K_{u} x_{u}(t) \\
& +\left(1-K_{a} A_{a}^{-1} B_{a}\right) u_{r}= \\
\hat{=} & -K_{a} x_{a}(t)-K_{u} x_{u}(t)+K_{r} u_{r}
\end{aligned}
$$

The control law is thus the sum of a linear weighting of the states $x_{a}$ and $x_{u}$ and a feed forward term from the driver's torque demand. It can be noted that no reference trajectories are needed for the evaluation.

\subsection{Linear Controller Properties}

The properties of the derived linear controller part are investigated using parameters obtained from experiments with a 25 ton Volvo FH16 heavy duty truck (see Appendix). Three transfer functions are first presented and then the influence of the performance index weighting factors is investigated.
As previously described, the controller objectives are to fulfill the driver's engine torque demand while on the same time damp driveline oscillations. This implies that the transfer functions from $u_{r}$ to $u$ and from $u_{r}$ to the output $y_{1}=\dot{T}_{\text {shaft }}$ are of interest. Oscillations can also be induced by road load variations which means that the transfer function from $T_{\text {load }}=v$ to $y_{1}$ is important.

$$
\begin{aligned}
g_{1}(s) & =K_{a}\left(s I-A_{a}\right)^{-1} B_{a} \\
G_{u_{r} u}(s) & =\frac{s K_{r}+K_{u}}{s\left(1+g_{1}(s)\right)+K_{u}} \\
G_{u_{r} y_{1}}(s) & =\left(C_{a} A_{a}\left(s I-A_{a}\right)^{-1} B_{a}+C_{a} B_{a}\right) G_{u_{r} u}(s) \\
G_{v y_{1}}(s) & =C_{a} A_{a}\left(s I-A_{a}\right)^{-1} B_{v}+C_{a} B_{v}
\end{aligned}
$$

Figure 5 shows the Bode diagram of the transfer function from the driver's input $u_{r}$ to the controller output $u$. The low frequency gain is unity and the torque demand is significantly damped in a frequency range around the resonance frequency. The dashed line shows that the high frequency gain is constant without prefiltering of the driver's torque demand. It is preferable to have a roll-off at high frequencies to reduce the noise sensitivity. The easiest way to achieve this is to introduce a low-pass prefilter for the driver's torque demand. The solid line shows the improvement with a first order filter with a time constant of $20 \mathrm{~ms}$. The same prefilter is used in the sequel.

The Bode diagrams in Figures 6 and 7 show that the resonance peak of the drive shaft torque derivative is well damped, both with respect to driver inputs and road load disturbances.

The controller has two tuning parameters $q_{1}$ and $q_{2}$, as presented in Section 3.2. $q_{1}$ influences the shuffle damping and $q_{2}$ affects the tracking of the driver's torque demand. Figure 8 illustrates the balance between them. These simulations are made using the control law (14) and assuming that the full state vector is measurable.

\subsection{Linear Controller Robustness}

The controller is tested for robustness by performing simulations on a set of models where a few parameters are varied, Figure 9. A torque limiting function is active during the initial torque rise, which limits engine torque to $1000 \mathrm{Nm}$. An anti-windup function that reduces the fourth state value when the controller demand is above the limit behaves sufficiently well. At $t=2 \mathrm{~s}$, the torque request is reduced and at $t=4 \mathrm{~s}$, an unmeasurable disturbance occurs. The controller manages to damp oscillations well.

\subsection{Backlash Traversal Controller}

The torque compensator handles engine torque variations and road disturbances well when the system is in the contact mode. But if the sign of the drive shaft torque changes, the 

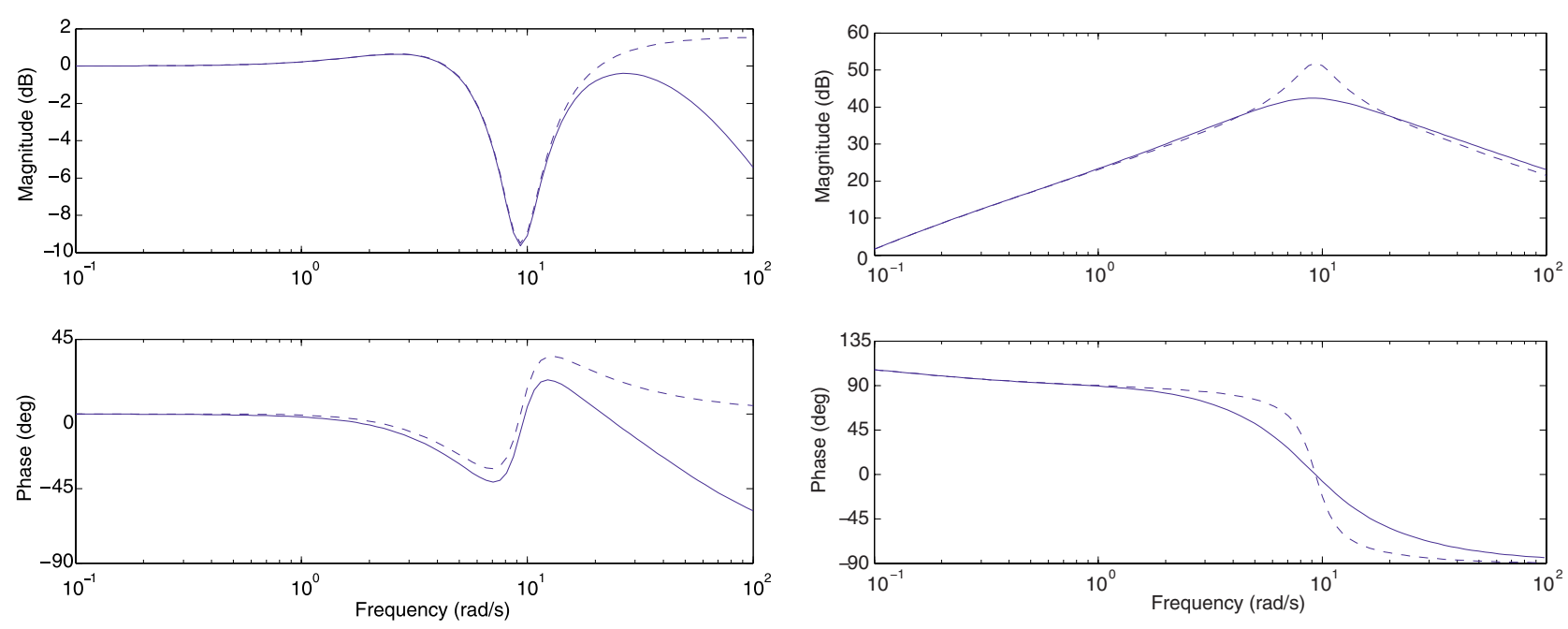

Figure 5

Bode plot of transfer function from the driver's torque demand to actual engine torque. The dashed line is without pre-filtering of the driver's torque demand and the solid line is with a first order low-pass prefilter with a time constant of $20 \mathrm{~ms}$.
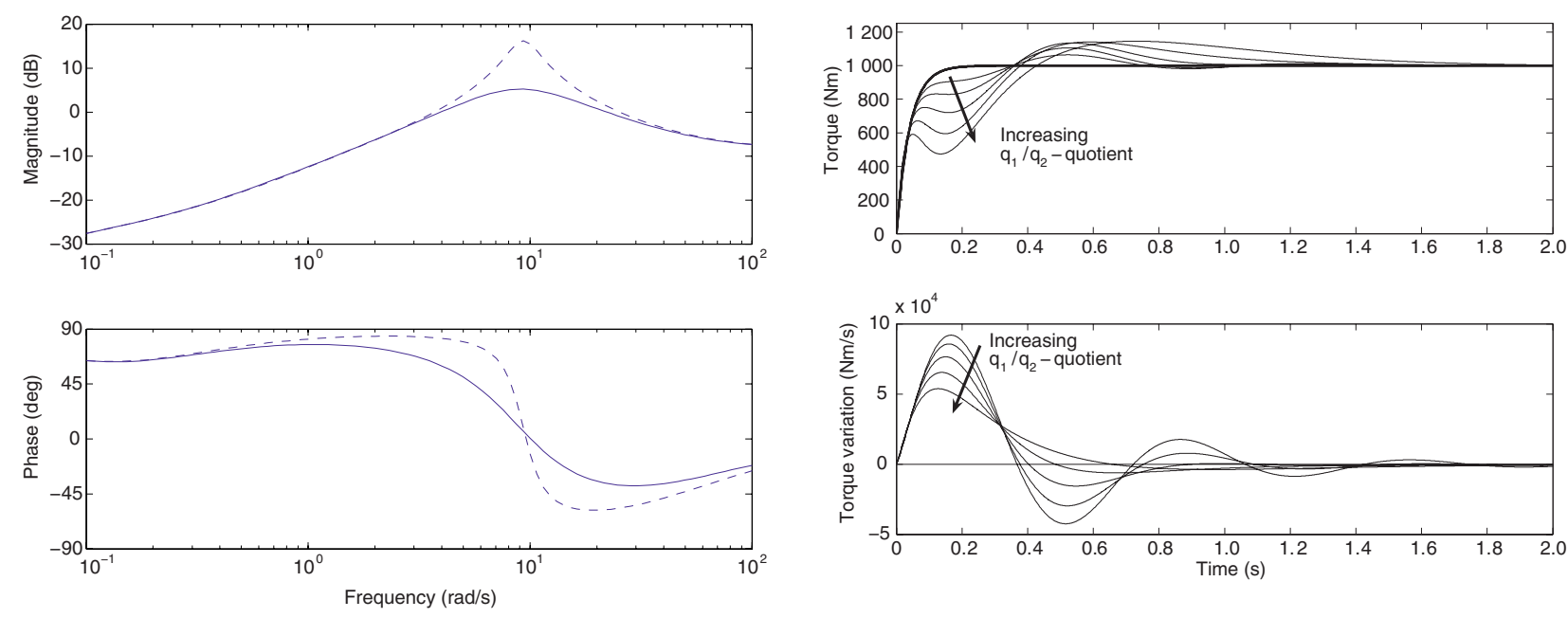

Figure 7

Bode plot of transfer function from road load to the derivative of the drive shaft torque. The dashed line is the characteristics of the system without controller, the solid line with controller.

Figure 6

Bode plot of transfer function from the driver's torque demand to the derivative of the drive shaft torque. The same low-pass filter as was used in Figure 5 is also used here to ensure a roll-off at higher frequencies. The dashed line is the characteristics of the system without controller, the solid line with controller.

\section{Figure 8}

The tuning parameters $q_{1}$ and $q_{2}$ affects the balance between tracking the driver's demand and damping of the shuffle. The solid line in the upper plot is the driver's torque demand and the thin lines are the controller outputs for a range of settings. A high shuffle damping results in large deviations from the driver's torque demand.

traversing of the backlash may cause shunts and amplification of the following oscillations as illustrated in Figure 3. The idea is to limit the torque request until the opposite side of the backlash is reached, so that the relative speed between the engine and the wheels is limited when the opposing side is reached. This is made by introducing a torque hold level that the requested torque may not exceed until contact is reached. 


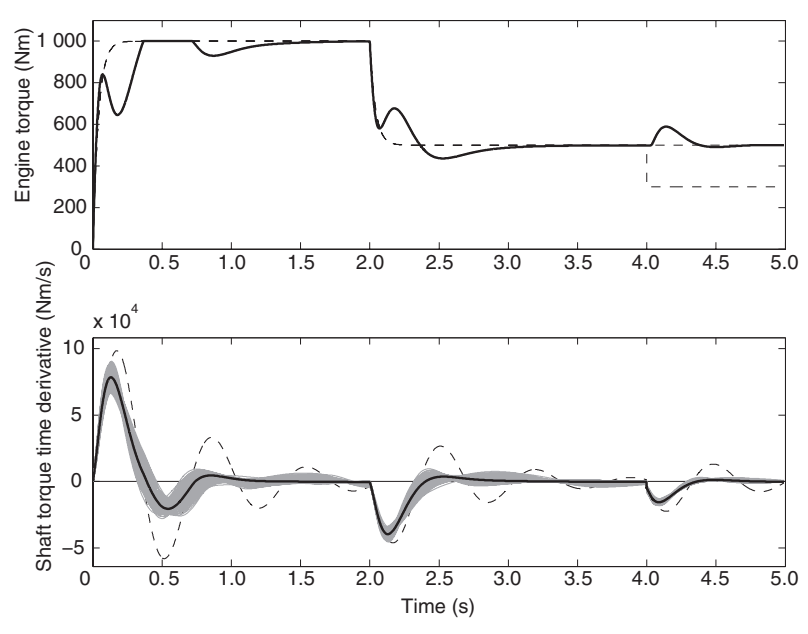

Figure 9

Simulation results for one controller setting (for a nominal plant model) and varying plant model parameters. The upper plot shows the reference torque request (dashed) and the actual engine torque (solid, thick). At $t=4 \mathrm{~s}$, an unmeasurable engine torque disturbance is introduced, which lowers the actual engine torque $200 \mathrm{Nm}$. The lower plot shows the time derivative of the drive shaft torque. The dashed line is the response to the reference torque request directly (without any regulator). The solid thick line is the closed loop behavior with the nominal plant model. The gray zone illustrates how the response varies for different plant models. The vehicle mass is varied $\pm 20 \%$, shaft stiffness is also varied $\pm 20 \%$ and the shaft damping is varied $\pm 40 \%$.
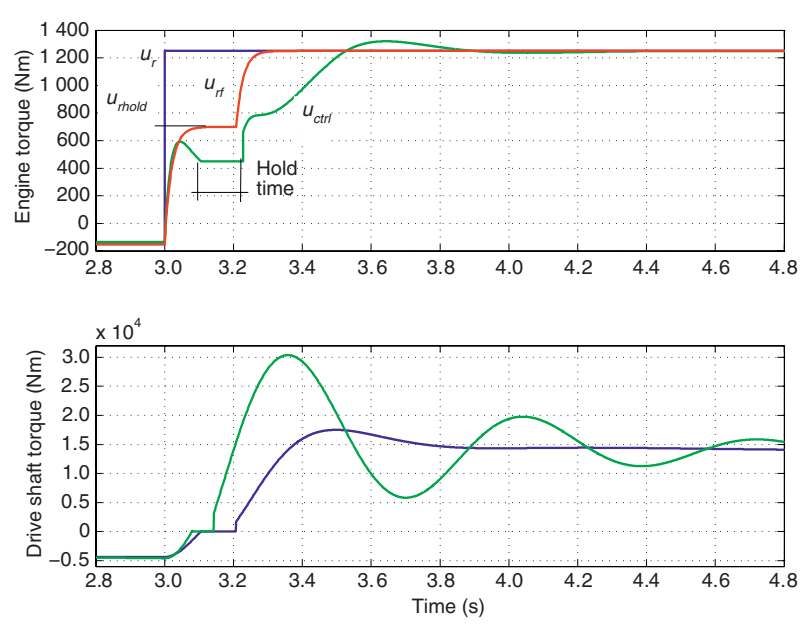

Figure 10

Simulation example of engine torque rise with backlash traversing.

Figure 10 shows a simulation example where a torque step rise is requested. The upper plot shows the reference torque $u_{r}$, the same signal slightly low-pass filtered $u_{r f}$ where also the hold level is visible. The third signal $u_{c t r l}$ is the controller output, i.e. actual engine torque demand. The
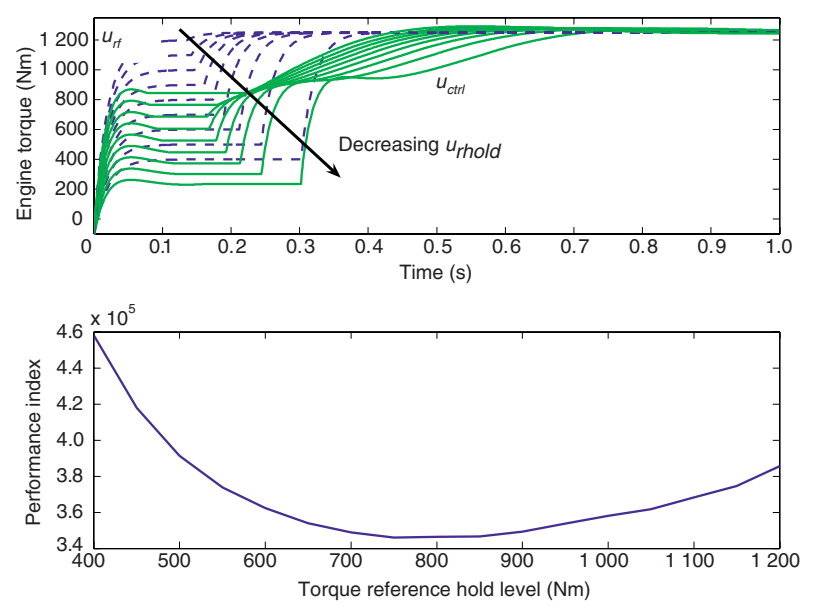

Figure 11

Engine torque optimization trajectories for different values of $u_{\text {rhold }}$ (upper plot) and corresponding loss function values.

lower plot shows the drive shaft torque increase, where the oscillatory curve shows the result without any controller and the smoother curve shows the outcome when the controller is active.

This behavior requires a modification of the performance index (4) to find an appropriate intermediate reference torque level that limits the jerk following the traversing of the backlash.

$$
J=\int_{0}^{t_{f}}\left(\frac{1}{2} y^{T}(t) Q y(t)+\frac{1}{2}\left(u(t)-u_{r}\right)^{2}\right) d t+q_{b} \dot{\theta}_{b}^{2}\left(t_{+}\right)
$$

The additional term is evaluated at the time instance $t_{+}$ which is defined as the time when the opposing side of the backlash is reached. The intermediate torque reference hold value $u_{\text {rhold }}$ is chosen to minimize (19) with the help of numerical optimization. During the optimization, the linear controller, that was derived previously, is active when the system is in contact mode. The controller output is held during the backlash transition. Figure 11 shows an example of how the controller output and the performance index (19) varies for a range of $u_{\text {rhold }}$-values and a reasonable $q_{b}$-value.

\subsection{Controller Tuning}

The tuning parameters of the controller summarize to $\left(q_{1}, q_{2}, q_{b}\right)$. The final tuning of these will be made in the vehicle to obtain the desired drivability. $q_{1}$ makes the tradeoff between controller responsiveness and how fast oscillations decay, $q_{2}$ is tuned to assure expected control value tracking of the driver's reference value. The effects of the shunt after the traversing of the backlash is determined by $q_{b}$. This gives the person responsible for the tuning a clear set of parameters to work with. The model based approach ensures the adaption to the specific vehicle. 


\section{EXPERIMENTAL RESULTS}

This section will present results from tests of the presented controller on a Volvo FH16 heavy duty truck. It is a $6 \times 4$ rigid truck, weighing 24.4 tons, with air suspension and a 16 liter $700 \mathrm{hp}$ engine with maximum torque $3100 \mathrm{Nm}$. It is intended for demanding long haul, including timber transports.

The tests were performed on a straight flat test road, driving at low speed $(10-20 \mathrm{~km} / \mathrm{h})$ on 4'th gear. The test road has smooth asphalt to avoid disturbances from the ground, which makes it easier to perform repeatable experiments.

\subsection{Test Setup}

The observer and control law are implemented on a laptop computer with CANalyzer-software, which is connected to the truck CAN-bus. During the tests, the accelerator pedal position is read by the laptop and converted to an engine torque demand via a pedal map (normally performed by the engine ECU). This engine torque demand is denoted $u_{r}$ and used in the control law (14), a linear combination of the observer states and a feed forward term from the driver's original torque demand. The control law output value (compensated driver's demand) is sent from the laptop to the engine ECU as a torque request with high priority, bypassing the ECU normal pedal handling. No modifications of the engine ECU software were needed.

The engine and wheel speeds are measured with the tooth wheels normally used by the engine controller and the brake system. These speed measurements are sent out on the CAN-bus in standard messages. The state observer also needs the engine torque as input. No torque sensor is however available, so the engine ECU estimation of the engine torque, which also is available on the CAN-bus, is used instead. This means that only the truck standard sensors are required by the controller.

The test setup means that communication delays are part of the closed control loop. These delays will not be present if the algorithm is implemented in the engine ECU, which is the preferred final location. This may be viewed as a robustness margin for the presented results.

\subsection{Model Parameters}

The success of the proposed controller relies on correct parameter values in the matrices (1) and (2). Some are considered known a priori, such as the engine inertia, friction, gearbox and the final gear ratios together with the wheel rolling radius. The vehicle mass and road load are estimated by another ECU, using information from the air suspension and an inclination sensor, and this information is available on the CAN-bus. The remaining parameters, the combined shaft stiffness $k_{s}$, the damping coefficients $c_{s}$ and $c_{w}$ and the backlash size $2 \alpha$, need to be estimated with experimental data. A methodology for estimating these parameters was described in [9]. The method is implemented on a laptop computer and uses only the input signals described in Section 4.1. The main parameters for the test vehicle are listed in Appendix.

\subsection{Observer}

The observer is the base for the evaluation of the control law and also for the switching of controller state between contact and non-contact mode, via the estimated drive shaft torque. A torque sensor would have been beneficial for the verification of the observer, but has not been available to the project. Test cases include mainly pedal tip-in and tip-out situations where the controller is expected to make significant compensations of the driver's torque demand.

Figure 12 shows a measurement of the observer behavior at an engine torque step down request, i.e. the vehicle accelerates when the driver suddenly releases the accelerator pedal. The observer is implemented using a zero order hold discretization. Initially, the vehicle is accelerating at high engine torque, $1200 \mathrm{Nm}$, up to the engine speed $1720 \mathrm{rpm}$. Zero engine torque is requested at $t \approx 14.2 \mathrm{~s}$. The drive shaft torque rapidly decays from $15 \mathrm{kNm}$ to $0 \mathrm{Nm}$ and the backlash is quickly traversed (the backlash angle goes from $+\alpha$ to $-\alpha$ ). As the drive shaft torque becomes increasingly negative (the first negative peak is $-13.5 \mathrm{kNm}$ ), the engine speed "swings back", until the drive shaft transmits zero torque and the backlash is entered again. The initial peak-topeak engine speed swing is $200 \mathrm{rpm}$. During the decoupled

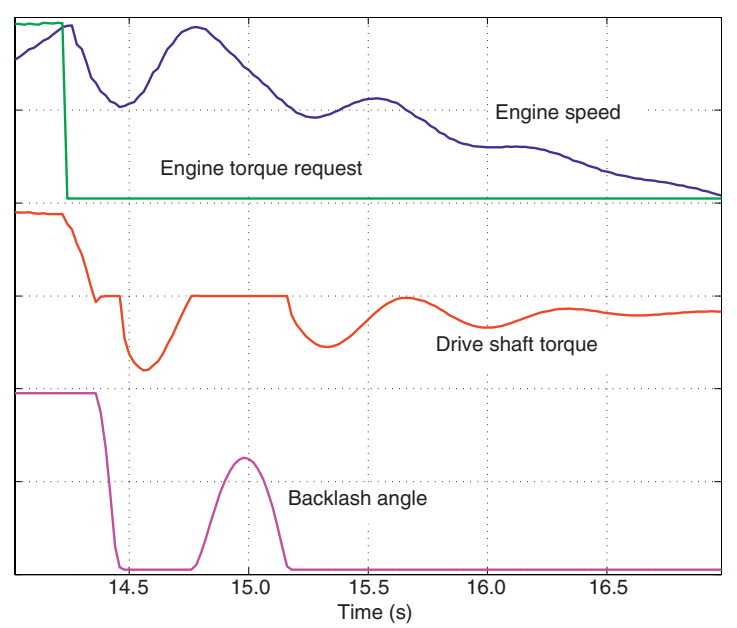

Figure 12

Observer example behavior at an engine torque down step request. The curve scales are normalized to give a qualitative view. The measured and the estimated engine speed signals are practically identical. The engine torque signal is estimated by the engine control unit and the drive shaft torque and the backlash angle are estimated by the combined observer. 

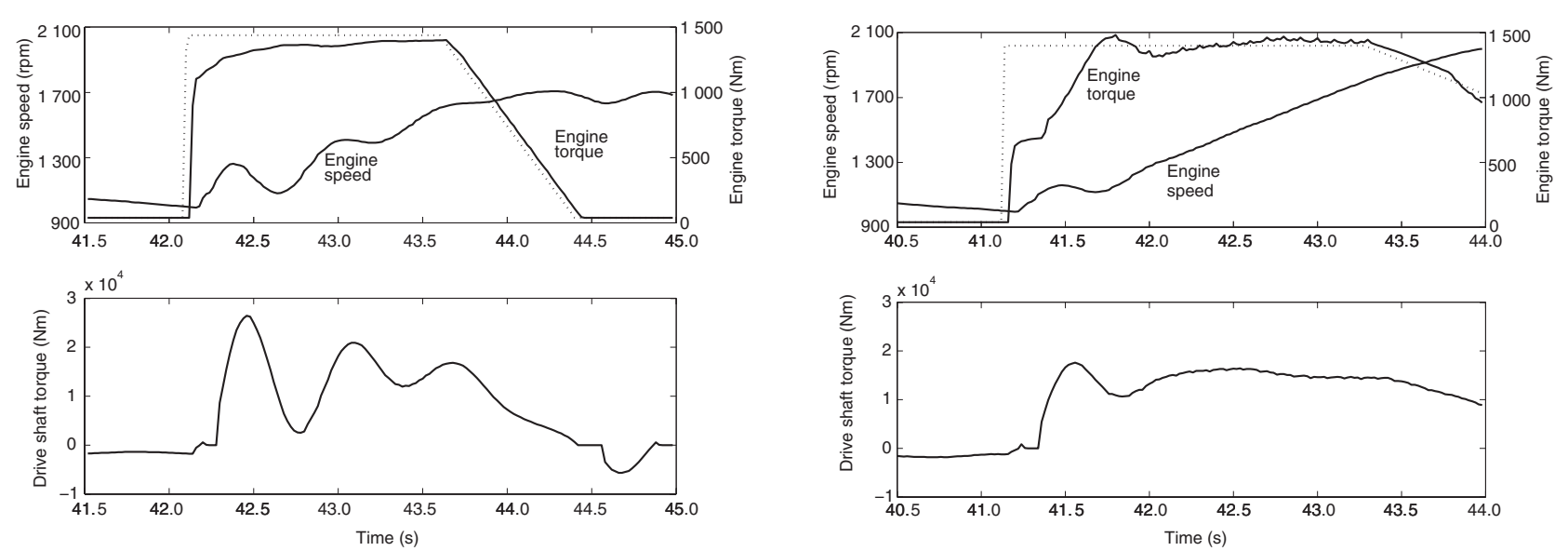

a)

b)

Figure 13

The figures show engine torque rise and engine speed measurements after a torque step request (torque request $=$ dotted line). The drive shaft torque (observer estimate) oscillates clearly less with the closed loop torque compensation. a) Without any damping control. b) With closed loop torque compensation.

period, the engine speed decays due to engine friction only. In this case, the position in the backlash never reaches the positive drive side before it falls back to the negative side again and thereafter stays there.

Unfortunately, neither measurements of the drive shaft torque nor backlash angle were available for verification of the observer accuracy. It can however be seen that the observer's drive shaft torque estimation is smooth and the switching between contact and non-contact modes appears to be consistent, without any fast transitions back and forth without apparent reasons. The measurement updates of the observer engine and wheel states are small. Indirectly, the observer performance is also verified by the good behavior of the closed loop controller in the experiments.

\subsection{Torque Compensator}

The implementation of the control law is straight forward from (14). The torque compensator is tested in the case when there is a stepwise request for a torque rise. The driveline is initially in contact mode on the negative drive side. Figure 13a shows a reference measurement of the engine speed following an engine torque step. Figure $13 \mathrm{~b}$ shows a corresponding step using the closed loop controller. The lower plot of the figures show the estimated drive shaft torque as estimated by the observer. The engine torque hold level $(750 \mathrm{Nm})$ during the backlash transition limits the shunt and the subsequent drive shaft torque peak. The following oscillations are quickly damped by the controller.
The torque compensator behaves well and in good agreement with simulations. The driver perceives a vehicle that quickly increases the tractive force, according to his demand, instead of causing uncomfortable oscillations when the accelerator pedal is depressed.

\section{CONCLUSIONS AND FUTURE WORK}

This paper presents a novel LQR-formulation of the well known driveline shuffle damping problem. The control law is derived and some properties are investigated in simulations. The controller is extended with an optimization based handling of the backlash transition that limits the shunt phenomenon. Finally, the results are verified by measurements in a heavy duty truck and the behavior looks very promising.

The implementation of the controller used for the tests was made on laptop that was connected to the truck's CANbus. This introduces communication delays between the measurements, control law evaluation and the actuation of the torque request. An implementation directly in the Engine Management System will avoid this and probably improve the controller performance. Future work will also involve an investigation on the possibility to create a mapping of the torque hold level during the backlash transition so that an on-line optimization is avoided.

\section{ACKNOWLEDGEMENTS}

This work was supported by Volvo Powertrain Corporation and Chalmers University of Technology. The project 
has been financed by The Program Board for Automotive Research (Vinnova) and Volvo Powertrain Corporation.

\section{APPENDIX - VEHICLE SPECIFICATION AND WEIGHTING FACTORS}

The simulation results and the control design are based on data obtained from system identification experiments with a Volvo FH16 Rigid $6 \times 4$ truck, Table 1 .

Simulations and experiments were performed with the loss function weighting factors $q_{1}=8 \cdot 10^{-5}, q_{2}=8$ and $q_{b}=4 \cdot 10^{5}$.

TABLE 1

Vehicle parameters

\begin{tabular}{c|c|c|c}
\hline Vehicle mass & 24450 & $\mathrm{~kg}$ & \\
Engine moment of inertia & 5.635 & $\mathrm{kgm}^{2}$ & \\
Wheel radius & 0.508 & $\mathrm{~m}$ & \\
Final gear ratio & 3.79 & - & \\
Gearbox ratio (4th gear) & 5.571 & - & \\
Combined shaft stiffness & 179 & $\mathrm{kNm} / \mathrm{rad}$ & (estimated) \\
Combined shaft damping & 8.26 & $\mathrm{kNm} /(\mathrm{rad} / \mathrm{s})$ & (estimated) \\
Combined wheel damping & 81.5 & $\mathrm{kNm} /(\mathrm{rad} / \mathrm{s})$ & (estimated) \\
\hline
\end{tabular}

\section{REFERENCES}

1 De La Salle S., Jansz M. (1999) Design of feedback control system for damping of vehicle shuffle, Proceedings of the EAEC conference, Barcelona.

2 Pettersson M., Nielsen L. (2003) Diesel engine speed control with handling of driveline resonances, Control Eng. Pract. 11, 319-328.
3 Bruce M., Egardt B., Pettersson S. (2005) On Powertrain Oscillation Damping using Feedforward and LQ Feedback Control, Proceedings of the 2005 IEEE International Conference on Control Applications - CCA2005.

4 Baumann J. et al. (2006) Model-based predictive anti-jerk control, Control Eng. Pract. 14, 259-266.

5 Berriri M., Chevrel P., Lefebvre D. (2008) Active damping of automotive powertrain oscillations by a partial torque compensator, Control Eng. Pract. 16, 874-883.

6 Fredriksson J., Weiefors H., Egardt B. (2002) Powertrain Control for Active Damping of Driveline Oscillations, Veh. Syst. Dyn. 37, 5, 359-376.

7 Templin P., Egardt B. (2009) An LQR torque compensator for driveline oscillation damping, Proceedings of the 2009 IEEE International Conference on Control Applications - CCA2009.

8 Lagerberg A., Egardt B. (2007) Backlash estimation with application to automotive powertrains, IEEE Trans. Control Syst. Technol. 15, 3, 483-493.

9 Templin P. (2008) Simultaneous estimation of driveline dynamics and backlash size for control design, Proceedings of the 2008 IEEE International Conference on Control Applications - CCA2008, pp. 13-18.

10 Nordin M., Gutman P.O. (2002) Controlling mechanical systems with backlash - a survey, Automatica 38, 1633-1649.

11 Glad T., Ljung L. (2000) Control Theory, Taylor \& Francis, Chapter 9.

12 Ferrari-Trecate G., Mehdi G. (2006) Observability analysis and state observers for automotive powertrains with backlash: a hybrid system approach, Int. J. Control 79, 5, 496-507.

Final manuscript received in May 2011 Published online in October 2011 